\title{
Structural Analysis of Factual, Conceptual, Procedural, and Metacognitive Knowledge in a Multidimensional Knowledge Network
}

\author{
Đurdica Vukić $\mathbb{D}$, Sanda Martinčić-Ipšić, and Ana Meštrovićc $\mathbb{D}$ \\ University of Rijeka, Department of Informatics, Radmile Matejčić 2, 51000 Rijeka, Croatia \\ Correspondence should be addressed to Ana Meštrović; amestrovic@uniri.hr
}

Received 2 July 2019; Revised 20 December 2019; Accepted 3 February 2020; Published 9 March 2020

Academic Editor: Roberto Natella

Copyright (C) 2020 Đurđica Vukić et al. This is an open access article distributed under the Creative Commons Attribution License, which permits unrestricted use, distribution, and reproduction in any medium, provided the original work is properly cited.

\begin{abstract}
Discovering the most suitable network structure of the learning domain represents one of the main challenges of knowledge delivery and acquisition. We propose a multidimensional knowledge network (MKN) consisting of three components: multilayer network and its two projections. Each network layer constitutes factual, conceptual, procedural, or metacognitive knowledge within the domain of databases as a standard course of computer science study. In the MKN layer, nodes are concepts or knowledge units and the edges are weighted with regard to Bloom's cognitive learning level. The projected network layers are contrasted with a monolayer network by comparing characterizations of the centrality measures: degree centrality, closeness centrality, betweenness centrality, and eccentricity. The study revealed indications of how concepts, supported with the higher number of previously introduced concepts, have a dominant role in knowledge acquisition, from a view of knowledge structure and content. The analysis of communities, assortativity coefficient, and overlap between MKN layers contributes to better structuring of knowledge. MKN enables systematic insights into the efficiency of knowledge integration across metacognitive layers, as well as the detection of crucial cognitive concepts that reduce/increase the cognitive load during learning.
\end{abstract}

\section{Introduction}

The organization of knowledge impacts outcomes in teaching and education, where much effort has been invested in the development of instructional strategies that may assist students in creating and organizing knowledge structures [1]. The representation of knowledge affects the learning processes and motivates the design of the teaching materials, especially in the e-learning systems [1-4]. Knowledge can be represented in the explicit form of a map (diagram), lattice, or network, where conceptual elements are interconnected, forming a cohesive and contingent system $[5,6]$. Then, the process of learning involves understanding what the key concepts are and how are they related, connected, and integrated into a more extensive system. Such interconnections between concepts have an essential role in establishing their meaning and affecting how concepts are introduced in teaching and how they are acquired in formal teaching and learning [2]. Cognitive load theory suggests that learners can absorb and retain information effectively only if it is provided in such a way that it does not "overload" their mental capacity [7]. Siew et al. in [8] review means of network science to provide a consistent and powerful approach to represent the structure and dynamics in cognitive systems, specifically in semantic memory and mental lexicon, hence revealing internal person's (or student's) representation. In this work, we investigate the possibilities of external representation of the knowledge in a selected domain and hence external representation in a system, i.e., e-learning system [9].

In educational settings, the use of graphical knowledge representation tools like concept maps has been a primary supporting technique for the formation of organized knowledge models. Consequently, concept maps have been applied at all levels of teaching, as well as in textbooks [1]. Since the amount of knowledge increases at an 
unprecedented pace, mapping activities align well with the processes of knowledge acquisition as they focus on integrating existing with new concepts [10]. Thus, through analyzing concept mapping difficulties that the learner encounters, there is an opportunity to learn, providing the cohesion and contingency of the relational structure of knowledge and enhancing long-term learning outcomes, retention, and transfer. Mapping of concepts into an interconnected structure is exceptionally efficient, in comparison to other representation techniques such as outlining or defining ideas, for learning about the relations between ideas [5]. Concept maps as knowledge integration tools elicit ideas as nodes (concepts) [11]. Therefore, analyses of the global structure of the interlinked key concepts can be conducted as the analysis of a complex network.

Research in [6] presented the idea of applying complex network theory and measures for the characterization of the concept maps in order to identify key concepts that provide the cohesion and contingency of the whole network of concepts. Motivated by research challenges addressed in $[6,8]$, in this study, we introduce the multidimensional knowledge network (MKN) based on the learning outcomes of crucial concepts in the domain. While the study of representing the structure in cognitive systems is mainly tasked with efficient internal representations of the mental model $[8,12-14]$, here we aim into the modeling of external knowledge which can be incorporated into an e-learning system of the domain or can be used to assess the student's progress in acquiring the subject, which is grounded in Bloom's taxonomy $[7,15,16]$.

The learning outcomes stem from different knowledge levels from Revised Bloom's taxonomy [16-18]. Revised Bloom's taxonomy differentiates factual, conceptual, procedural, and metacognitive knowledge dimensions. Factual knowledge captures discrete, isolated content elements (terminology and knowledge of specific details and elements). Conceptual knowledge comprises classifications and categories, principles and generalizations, and theories, models, and structures of concepts. Procedural knowledge includes skills and algorithms, techniques and methods, as well as knowledge of the criteria used to determine and justify "when to do what" within specific domains and disciplines. Metacognitive knowledge encompasses strategic knowledge; knowledge about cognitive tasks, including contextual and conditional knowledge; and self-knowledge [7].

In this work, we study the principles of representing and analyzing how domain knowledge (concepts) can be modeled across four knowledge levels in a complex network framework aiming at the facilitation of knowledge modeling, acquisition, and transfer performance.

We propose a multidimensional knowledge network $(\mathrm{MKN})$, which is based on the multilayer network. Multidimensional knowledge network is composed of three parts: (i) directed and weighted multilayer network, (ii) interlayer projection of multilayer network, and (iii) monolayer projection of multilayer network. The interlayer projection is constructed by projecting the edges between layers to the higher layer in the hierarchy, while the monolayer projection is constructed by projecting all nodes and edges onto one layer. This way the defined model enables systematic insights into the knowledge dimensions and efficiency of knowledge integration across metacognitive layers, as well as the detection of key cognitive concepts that reduce/increase the cognitive load in processing information. The results obtained from MKN analysis can shed light on the causes of increased cognitive demands, indicate vulnerabilities in the knowledge (more specific and identify knowledge units that require modification of instructional strategies), and consequently guide the design and optimization of learning outcomes. For the experimental purposes, we evaluate proposed MKN in the field of computer science-specifically for the databases, including standard topics in database design and implementation. For the domain of "database," we construct the multidimensional knowledge network, following the set of learning outcomes for the database course and provide a detailed analysis of key concepts in the proposed MKN model.

In the second section, we describe the theoretical background of network science and cognitive complex networks. Moreover, we give an overview of network theory applications in cognitive science and knowledge representation and their contributions. The third section provides the methodology for multilayer network analysis with the definition of qualitative and quantitative measures. The fourth section covers the gathering of experimental data and network construction principles for database-domain learning outcomes. The fifth section is dedicated to the reporting and discussion of the MKN model. The paper concludes in the sixth section with future research plans.

\section{Background}

The emergence of a new paradigm-complexity lies at the core of the information age, reflecting everything that has intrinsically complex behavior and cannot be described in a comprehensive manner [19]. The ability to reason and comprehend such properties finds its roots in graph theory and sociology when Paul Erdős and Alfréd Rényi research study draw a new multidisciplinary interest into the study of complex networks [19-21]. The rise of interest in understanding general properties of complex systems had an impact on a substantial upsurge in the study of interconnected structures in many disciplines and, generally, the evolution of network science [8, 21-33]. The analysis of the immense amount of data, due to its exponential growth, resulted in novel analytic methods for complex network analysis considering global and local network structures as well as the time-varying and multilayer nature. Multilayer networks explicitly incorporate multiple channels of connectivity and constitute the natural environment to describe systems interconnected through different categories of connections: a layer represents each channel (relationship, activity, and category) and the same node or entity may have different kinds of interactions (different set of neighbors in each layer) [31, 34, 35]. Our research in the direction of multiple layers' analysis is motivated by the fact that multilayered network structure is fundamentally more 
expressive than individual layers [36]. They use multiplex networks for the task of predicting the ordering with which words are acquired. However, the similar argumentation and motivation hold in the case of the learning process as well. Furthermore, multilayer analysis allows quantification of distinct phases of learning and multilayered networks.

\subsection{Related Work on Conceptual and Cognitive Complex} Networks. Individuals differ in their ability to learn from experience, to adapt to new situations and overcome challenges, to understand simple or complex ideas, to solve realworld or abstract problems, and to engage in different forms of reasoning and thinking $[5,8,17,37,38]$. Knowledge acquisition and integration activities are designed to help learners construct a more coherent understanding by developing criteria for the ideas that they encounter. Concept maps as knowledge integration tools elicit knowledge elements as nodes (concepts) [5,11] and relations between them as edges. Thus, the concept maps are network representations of the organization of the concepts. The advantage of such representations emphasizes the relational structure of knowledge, where the concepts and principles are interconnected and where the principles of making the connections can be explicated [39]. Concept mapping is exceptionally efficient, in comparison to other techniques such as outlining or defining knowledge elements [5].

Schwendimann in [11] indicates that concept maps can support knowledge integration processes by eliciting core ideas and connections and making possible clusters or hierarchies visible. Similarity, the authors in [5] report that graphic organizers, such as concept maps, can foster the integration of fragmented ideas toward an organized interconnected network of ideas. Moreover, recent research study focusing on the structure of the concept map suggests that better understanding and the high quality of students' knowledge are reflected in interconnected and web-like structures [40]. The authors in [41] highlighted the role of cognition and connection to language complex network by the principle that human performance can be related to network properties, further suggesting that network properties might provide evidence of or have an influence on human cognition as related to the acquisition of language. Gurevych in [42] proposed a method to generate artificial definitions of concepts from a conceptual network of words, finding that semantic relatedness of words compensates the lack of definitions in a conceptual hierarchy by generating a textual definition of the concept automatically from a knowledge base. The semantic relatedness metric generated glosses that correlate very well with human judgments of semantic relatedness. In [39], the authors state that, in practice, successful instruction and learning, which set its goals on the holistic understanding, require a deeper understanding of the knowledge as a coherent and connected structure. Consequently, the coherence and connectivity of such a knowledge representation are known to be closely related to knowledge production, acquisition, and processing.

Knowledge processing and acquisition have been described in the framework of complex networks theory
$[22,27,43]$. The complex network methodology is well suited for a description of relations in the conceptual knowledge and dynamics of the retrieval process of knowledge [8]. Network motivated approaches are well adapted to the related problems of knowledge modeling, retrieval, and acquisition. Reported research study, in general, is indicating the importance of establishing connections between words regarding their meaning, semantic relations, phonological similarity, or syntax $[2,8,13,27,44]$. Hence, a lot of knowledge-related studies are intertwined with studies of language complex networks [12, 35, 45-48]. For instance, the authors in [49] consider the lexical structure of topics in a course as a monolayer lexical network of terms. The study reveals that extension to deeper contextual levels, by the inclusion of more remote connections between the terms (although in the same layer), facilitates the representation of knowledge and concludes that methods are sensitive enough to lexical or semantic features of the text.

Traditionally, researchers $[1-4,6,34,35,39,42,49-53]$ analyze isolated aspects of the network structures such as the number of links to concepts, (degree) [51], the number of components (unconnected parts of the content structure), the subgraph measures, and communicability betweenness centrality measures to derive rankings of different nodes based on how important each node is in providing cohesion and contingency [6]. A study in [51] is based on degree, clustering, transit efficiency, betweenness, and closeness centrality measures for analysis of the content structure of video lessons and identification of the key aspects of content structure concerning student learning gains. Siew in [50] analyzed monolayer conceptual networks from the concept maps generated by students of psychology and confirmed that concept networks differed across students and predicted learning outcomes. In short, the study concluded that concept networks with larger average shortest path lengths were associated with higher scores, hence suggesting that network science can be used to quantify the conceptual structure of a learner's knowledge. The common shortcoming of listed work is the granularity of the examined information since only single and isolated aspects of the knowledge are investigated in a monolayer setup.

Cognitive complex networks establish the foundation for understanding the principles for the study of conceptual networks in a more comprehensive manner. The aim for understanding the cognitive processes behind knowledge construction and its acquisition leads to the development of integral computational models for cognitive processes in learning [2]. Hence, they reach beyond monolayer networks into multilayered or multiplex structures. Research on cognitive networks utilizes the framework of multiplex lexical networks for investigating lexical retrieval from memory [13]. It uses the multiplex network to study how the layout of word-word similarities in the mental lexicon can lead to priming effects on multiple combined semantic and phonological levels. Multiplex lexical networks have proven fundamentally more powerful in investigating the process of early word acquisition $[13,36,46]$ and for detection of the core structure of mental lexicon, indicating the significance 
of integrating the importance of multiple word-word relations [14]. The conceptual network of the English language in [54] has also been examined through the means of cognitive science, wherefrom the standpoint of retrieval of information from associative memory, the small-world property of the network represents a maximization of retrieval efficiency.

In this work, we are aiming to fill the gap of the structural representation of the knowledge organized according to revised Bloom's taxonomy into factual, conceptual, procedural, and metacognitive knowledge employing layers in a multilayer complex network.

\section{Methodology for Multilayer Network Analysis}

The study of complex systems has impelled researchers to move from simple graph representations to more abstract analyses by including multiple subsystems and layers of connectivity [42]. Different notions of multilayer networks can be obtained with regard to various constraints, which give rise to networks of networks [26], multidimensional networks [28, 55], multilayer networks [29, 32], multiplex networks $[13,24,36,45,46,56,57]$, interacting networks [25], interdependent networks [58], and many others that have been introduced [34]. A theoretical framework of multilayer network structures from the literature addresses a general form of multilayer network [31, 34].

3.1. Multidimensional Knowledge Network Model. In this research, we propose a novel, integrative model for knowledge representation that enables a multidimensional analysis. The proposed model is based on the multilayer network with two extensions. The first extension is defined as the interlayer projection of the initial multilayer network, which is constructed by projecting interlayer edges onto one layer according to a predefined rule. The second extension is defined as the monolayer projection of the initial multilayer network, which is constructed in a way that all nodes and edges are projected onto one single layer.

In this section, we give definitions of all these formal models, and in the next section, we provide a context and interpretation of defined models.

According to [34], a multilayer network is defined as a pair:

$$
M=(G, C)
$$

where

$$
G=\left\{G^{a} ; \alpha \in\{1, \ldots, M\}\right\}
$$

is a family of networks (graphs) $G^{a}=\left(V^{\alpha}, E^{\alpha}\right)$ called network layers of $M$ and

$$
C=\left\{E^{\alpha \beta} \subseteq V^{\alpha} \times V^{\beta} ; \alpha, \beta \in\{1, \ldots, M\}, \alpha \neq \beta\right\},
$$

is the set of interconnections between nodes of different layers $G^{\alpha}$ and $G^{\beta}$ where $\alpha \neq \beta$.
Layers are annotated as numbers from the set $\{1, \ldots, M\}$, where $M$ is the number of layers. The network, multilayered as well, can be directed or undirected and weighted or unweighted; however, the selected network model has to be consistent for all layers in the multilayer network [35]. Therefore, the whole multilayer network can be defined as directed or undirected and weighted or unweighted. In this research, we construct a weighted and directed multilayer network.

The set of nodes of the network layer $V^{\alpha}$ is denoted by $V^{\alpha}=\left\{x_{1}^{\alpha}, x_{2}^{\alpha}, \ldots, x_{N_{\alpha}}^{\alpha}\right\}$, and the adjacency matrix of each layer $\alpha$ is denoted by $A^{[\alpha]}=\left(a_{i j}^{\alpha}\right)$, where

$$
a_{i j}^{\alpha}= \begin{cases}1, & \text { if }\left(x_{i}^{\alpha}, x_{j}^{\alpha}\right) \in E_{\alpha}, \\ 0, & \text { otherwise }\end{cases}
$$

for $1 \leq i, j \leq N_{\alpha}$, and $1 \leq \alpha \leq M$. The interlayer adjacency matrix corresponding to $E^{\alpha \beta}$ is the matrix $A^{[\alpha \beta]}=\left(\alpha_{i j}^{\alpha \beta}\right)$ defined by

$$
a_{i j}^{\alpha \beta}= \begin{cases}1, & \text { if }\left(x_{i}^{\beta}, x_{j}^{\beta}\right) \in E^{\alpha \beta}, \\ 0, & \text { otherwise. }\end{cases}
$$

Note that, in the case of weighted multilayer network, the adjacency matrices contain corresponding weights instead of 1 , denoted as $A^{[\alpha]}=\left(w_{i j}^{\alpha}\right)$ in the case of intralayer connections and $A^{[\alpha \beta]}=\left(a w_{i j}^{\alpha \beta}\right)$ in the case of interlayer connections. Additionally, we define and consider a special type of directed multilayer network, which can be derived from the initial multilayer network by projecting interlayer edges onto one single layer according to the target node.

For a given directed multilayer network $M$, we define an interlayer projection of $M$ denoted as

$$
i p(M)=\left\{G_{i p}^{\alpha} ; \alpha \in\{1, \ldots, M\}\right\},
$$

in a way that, for every edge from the set of interlayer connections, $e_{k}^{\alpha \beta}=\left(x_{i}^{\alpha}, x_{j}^{\beta}\right) \in E^{\alpha, \beta}$ and we shift the starting node $x_{i}^{\alpha}$ to the layer $\beta$; hence, it becomes the node $x_{i}^{\beta}$. Accordingly, the existing edge $e_{k}^{\alpha \beta}$ is projected to the $\beta$ layer in the way that it becomes a new edge: $e_{k}^{\beta}=\left(x_{i}^{\beta}, x_{j}^{\beta}\right) \in E^{\alpha}$. The result of the projection is a family of networks $\left\{G_{i p}^{\alpha} ; \alpha \in\{1, \ldots, M\}\right\}$ without interlayer edges. The projection rule assures that all interlayer edges are projected onto the target layer, including the projection of a starting node $x_{i}^{\alpha}$ to $x_{i}^{\beta}$. In the continuation of the paper, we will refer to the interlayer projection of $M$ as projection of $M$ or multilayer projection (MKN projection) in short.

Next, we define the monolayer projection of the initial multilayer network $M$, denoted as $m p(M)$, in the way that we project all nodes and links from all $M$ layers to one single layer. In the continuation of the paper, we refer to that network as a monolayer (projection) network in short. In Supplementary Material, we list the definition of all network measures used for the quantification of a multilayer network, a multilayer projection network, and a monolayer network.

Finally, the multidimensional knowledge network is defined as a triple:

$$
M K N=(M, i p(M), m p(m)) .
$$


3.2. Modeling the Network of Concepts. Given the fact that the network of concepts, in its' simplest definition, represents a system of connected parts, we assume that the knowledge system of any domain (subject) can be modeled as a complex network of knowledge. In the context of the selected domain (course), the concepts are nodes, and their relationships may be represented as edges that connect knowledge units. The edges are directed, reflecting the dependence of a hierarchical ordering that follows from the navigational path of learning in which one unit of knowledge is introduced (acquired) before the other. Course design reflects an effective instructional plan, aiming to optimize alignment between learning objectives, assessments, and instructional activities organized in a specific ordering of topics (i.e., crucial concepts of the domain). Hence, the assumption is that the network of concepts follows the ordering of efficient knowledge acquisition through the learning process $[1-4,6,39,49]$.

Determining learning outcomes start from a higher level and, at first, determines the learning outcomes for the study program, then for the module, and then for the group of subjects, followed by outcomes of individual subjects which are finally decomposed into knowledge units $[17,59]$. The planned outcomes (learning objectives) can be expressed in terms of the content (expressed as nouns) and the cognitive process needed (expressed as verbs). In this sense [7], the original Bloom's taxonomy was one-dimensional because the categories contained only nouns (objects) and verbs (actions). The verb generally refers to the actions associated with the intended cognitive process. The object generally describes the knowledge students are expected to acquire or construct. The cognitive process dimension represents a continuum of increasing cognitive complexity-from remember to create [16]. Each node is a concept or knowledge unit, and it is a semantic part of a learning objective. The relation between two nodes (two knowledge units) is established if these two knowledge units appear in the same (common) learning objective (outcome), and vice versa; the learning objective is a relation between two concepts (nodes) concerning the cognitive process and the knowledge dimensions according to revised Bloom's taxonomy. Revised Bloom's taxonomy differentiates between four dimensions of knowledge: factual, conceptual, procedural, or metacognitive knowledge [16]. According to this taxonomy, each level of knowledge corresponds to the level of cognitive process (load), so a student can remember factual or procedural knowledge, understand conceptual or metacognitive knowledge, or analyze metacognitive or factual knowledge [15-18]. Moreover, according to the revised version of Bloom's taxonomy, there are six dimensions of the cognitive process: remembering, understanding, applying, analyzing, evaluating, and creating.

Hence, revised Bloom's taxonomy can be represented as a multilayer network, where each layer models one dimension of knowledge. To this end, we propose a multidimensional knowledge network (MKN), formally defined in the previous section.

First, we define a multilayer network $M$, which consists of four layers. Each layer represents one dimension (the knowledge level) of Bloom's taxonomy: factual, conceptual, procedural, and metacognitive knowledge. In each layer, nodes represent knowledge units, defined according to the learning outcome. It is important to note that one node may belong to different layers since it can be associated with different learning outcomes-hence different levels of Bloom's taxonomy. Still, it is not required that all nodes (knowledge units) are present on all layers; i.e., some concepts are, for instance, present at factual and conceptual layers and not at procedural and metacognitive ones. Therefore, we opt for modeling of the multilayer and not for the multiplex network.

Nodes are connected between each other in one layer (intralayer edges) and across layers (interlayer edges). An edge between two nodes (knowledge units) is constructed if there is a learning outcome in which the first knowledge unit is connected to the second knowledge unit (in terms of sequence which follows the order of knowledge acquisition within the domain). The edges are weighted with regard to Bloom's cognitive process dimensions, and weights of the corresponding edge depend on the cognitive learning level to be achieved through the learning process that includes two nodes (knowledge units). Hence, remembering receives a weight of 1 , understanding 2 , applying 3 , analyzing 4 , evaluating 5 , and creating 6 .

Finally, we also construct the monolayer projection of the multilayer network $M$. Hence, a monolayer network contains all nodes and edges, weighted with respect to the cognitive process dimension and analyzed as a classical complex network providing information about the global network properties. Results of monolayer network analysis represent "initial raw" data that can be used to identify which top-ranked (monolayer) nodes, according to differences in results of monolayer and multilayer analysis, should be reviewed concerning its knowledge dimension identity in multilayer and projected components of the MKN. For example, high in-degree nodes in a monolayer network should indicate nodes at the factual knowledge dimension since it refers to isolated facts and details of concepts at the very early stage of learning. In the continuation, the monolayer network is compared to projected layers in order to quantify the specific properties of each layer. This is grounded in the theory of learning. When the units of the task are being processed simultaneously or when the content has a high degree of interactivity among elements, the high cognitive load will be imposed on the student, even when the number of interreacting elements is relatively small. As the intrinsic cognitive load is essential for the achievement of specific learning goals (understanding of the problem, construction of higher structures of knowledge, and their flexibility), it must be within the capacity of working memory (i.e., within limits of intrinsic cognitive load).

Figure 1 (adapted from [16]) illustrates an edge construction rule for the learning outcome. "Students will differentiate between terms database and DBMS." This learning outcome results with two nodes (knowledge units): database and DBMS with a verb differentiate, and establishes the intralayer edge in 4th (analyze) cognitive process dimension on the factual layer. Similarly, for the outcome: 


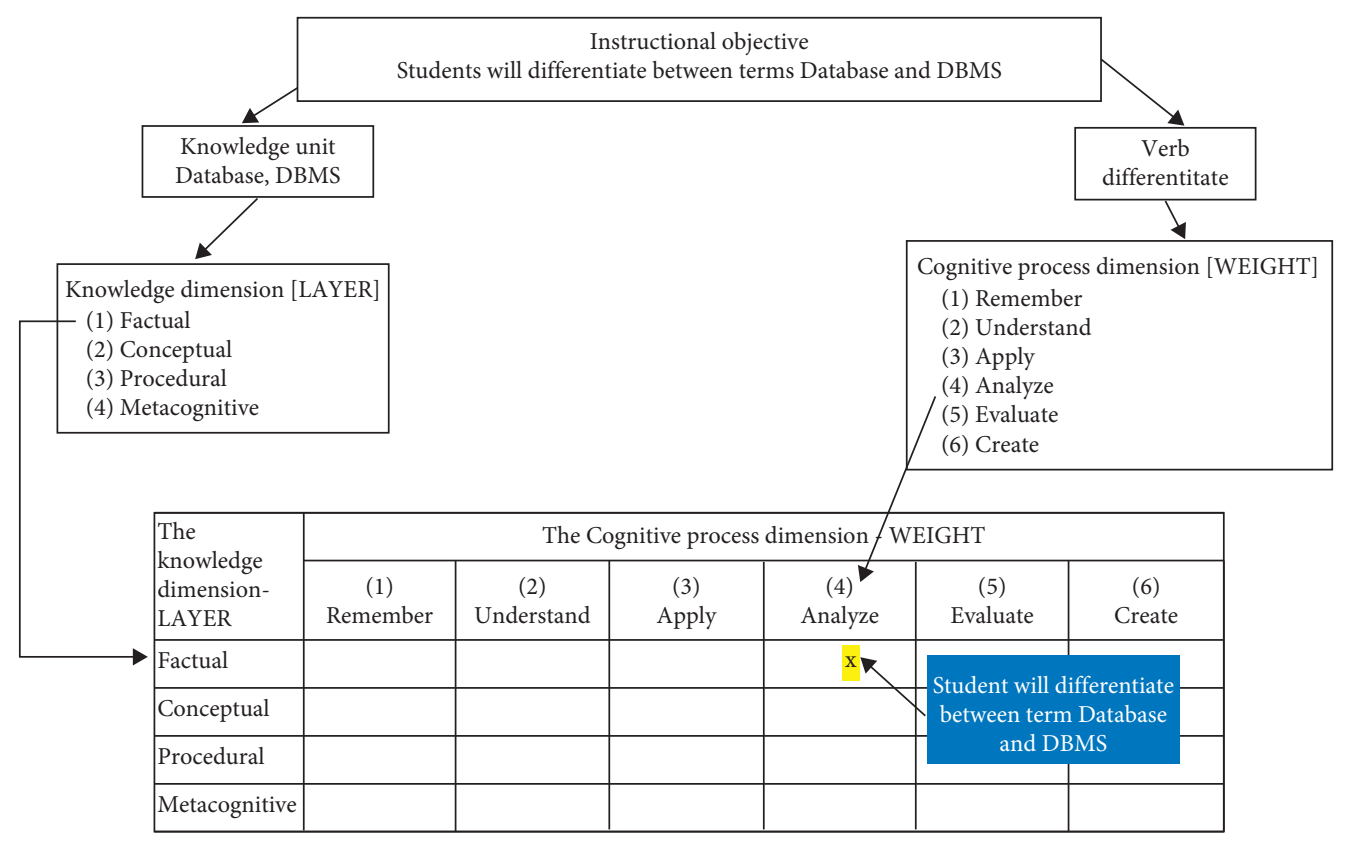

Figure 1: Construction of edges for the outcome: "Students will differentiate between terms database and DBMS" results with two nodes (knowledge units): database and DBMS and the verb differentiate establishing the edge with weight 4 in cognitive process dimension (analyze) at the layer of factual knowledge.

"Student will explain the correlation between $4 \mathrm{NF}$ and Multivalued dependency." We define interlayer edge: (1) node- $4 \mathrm{NF}$ at procedural layer and (2) node-multivalued dependency at conceptual layer with weight 2 (verb: understand). It is worth noticing that knowledge units are assumed to lie along a continuum from concrete (factual) to abstract (metacognitive). The conceptual and procedural categories overlap in terms of abstractness. The verb generally describes the intended cognitive process, and the concept generally describes the knowledge students are expected to acquire [16].

The process of the construction of the multidimensional knowledge network MKN with all three components $M, i p(M)$, and $m p(M)$, and the process of the analysis can be summarized in the following ten steps. Formalization of the process in ten steps can serve as a recipe for the transfer of the proposed methodology into a new domain.

Step 1. Selecting the knowledge domain and defining a hierarchical list of concepts (knowledge units of the instructional plan for domain). For instance, if we decide to construct the MKN for the database domain, we will define concepts like tables, relations, normal form, and index.

Step 2. Designing relevant learning outcomes from relations between concepts and aligning them with respect to Bloom's taxonomy of cognition. For example, from the learning outcome, "Students will explain the use of database normalization as the systematic approach of decomposing tables." we can derive a relation: decomposing (tables and database normalization).

Step 3. Constructing the first component of the MKN, a multilayer network $M$ : again nodes are concepts from the domain and one node can belong to one or more layers according to the learning outcomes that include that concept.

Step 4. Constructing the second component of the MKN, an interlayer projection of $M$, where we project the interlayer edges into the target layer, as defined in Section 3.1. As the result, we have ip $(M)$, which consist of four network layers, without interlayer connections.

Step 5. Constructing the third component of the MKN, a monolayer projection of $M: m p(M)$, where we project all nodes and edges onto one single layer. As the result, we obtain $m p(M)$, which consists of one network layer with all the nodes and edges.

Step 6. Analyzing and comparing all network layers defined in $i p(M)$ and $m p(M)$ on the global level.

Step 7. Analyzing and comparing all network layers defined in $i p(M)$ and $m p(M)$ on the local level in terms of identifying key concepts on each layer.

Step 8. Identifying communities aiming for the justification of knowledge organization and hierarchical partitioning of instructional content into coherent groups of concepts.

Step 9. Analyzing four layers in terms of assortativity mixing.

Step 10. Analyzing four projected layers in terms of node and edge overlapping. 


\section{Multidimensional Knowledge Network Construction for the Learning Outcomes in the Database Domain}

The concepts and their relationships are established from knowledge units defined in database syllabus standardly included in the computer science bachelor study (steps 1 and 2-please note that two authors have a background in teaching relational database subjects and acted as experts in the domain). The process continues with the construction of a multilayer knowledge network for the database domain (Step 3). First, we construct the multilayer network $M$. The multilayer network has a factual, conceptual, procedural, and metacognitive layer. Each node is a unit of knowledge, and it is a semantic part of a learning objective. Two nodes are connected if there is a learning outcome that includes both units. The multilayer network is constructed as directed and weighted network of concepts.

Next, we construct a projection of a multilayer network (Step 4). The projected network is directed and weighted and has factual, conceptual, procedural, and metacognitive layers. The factual layer contains 19 edges/25 nodes, the conceptual layer 57/43, the procedural layer 50/42, and the metacognitive layer 20/25 edges/nodes. Figure 2 presents four projected layers of a multilayer network $M$ for the learning outcomes of the database domain. Layer with factual cognitive knowledge is on the left, followed by layers of conceptual and procedural knowledge, while the metacognitive layer is at the rightmost position. Then, we construct the monolayer projection (Step 5), again as directed, and weighted network with 59 nodes and 147 edges.

Next, we analyze the projected networks on the global level (Step 6). The analysis is based on the quantification of standard network measures as defined in Supplementary Material. For both projected multilayer and projected monolayer networks, we calculate average degree, average weighted degree, network diameter, average path length, average clustering coefficient, graph density, and the number of connected components.

Global level quantification is followed by local level quantification of network properties (Step 7). The first aim of the analysis is to determine which concepts are the key ones that stimulate cognitive processes and are of importance for effective knowledge acquisition. According to the results obtained in our previous study on keywords extraction $[34,35]$, initially, we use degree, in-degree, and out-degree (also with weighted variants) and proceed with centrality measures of closeness, betweenness, and eccentricity.

Then, we analyze communities (Step 8), aiming for the justification of knowledge organization and hierarchical partitioning of instructional content into coherent groups of concepts.

Next, we compare multilayer characterizations of assortativity mixing in terms of Pearson and Spearman correlations between layers for assessing the resemblance and coherence between Bloom's knowledge dimensions (Step 9).
And finally, we perform the overlapping analysis (Step 10), which enables better insights into relatedness of four projected layers in terms of node and edge overlapping.

All visualizations and network measures are obtained with Gephi [60] and MuxViz [30] tools. Gephi is opensource software for graph and network analysis, which comes with a range of layout algorithms [60]. MuxViz is a free and open-source package for the analysis and visualization of multilayer networks [61].

\section{Results and Discussion}

In this section, we represent the results of the systematic analysis of network layers on the global, local, and meso-scale level and provide the results of measuring of correlations and overlapping between MKN layers. The measures have been selected to reveal crucial structural properties: identification of central concepts in $\mathrm{MKN}$, detection of a coherent cluster of concepts, and quantifying the relationship between different levels of the abstraction in the domain. Moreover, the central open questions are to understand the hierarchical dependencies along the knowledge dimension and dependency between knowledge units.

5.1. Analysis of the MKN Network Layers on the Global, Local, and Meso-Scale Level. Initially, we present the results of global characterization of the structural properties for projected multilayer and monolayer of the multidimensional knowledge network (MKN) in Table 1. All global measures (average degree, average weighted degree, network diameter, average path length, average clustering coefficient, and network density) are calculated for the largest connected component. Note that equations and explanations of all these network measures are given in the Supplementary material.

Global network measures enable only the coarse differentiation between layers. Still, we can notice that the conceptual and procedural layers exhibit similar properties-higher values of average degree, average weighted degree, diameter, average path length, and graph density in comparison to factual and metacognitive layers. Moreover, conceptual and procedural layers exhibit properties that are closer to the monolayer network than to the other two layers. The average clustering coefficient has low values for all four layers (for the metacognitive layer, the clustering coefficient is so low that it was not possible to calculate it). The monolayer network has a higher clustering coefficient that may indicate a higher cognitive load during the learning of new and yet strongly interwoven concepts, which supports the initial premise of balancing the cognitive load during learning, staring from concrete to abstract, and betterinterconnected knowledge units of the domain.

Moreover, factual and metacognitive layers have more than double the number of components compared to conceptual and procedural layers, which reflects in the higher values of global network measures. The number of connected components is the highest in the factual layer, and together with smaller network diameter is the reflection of 


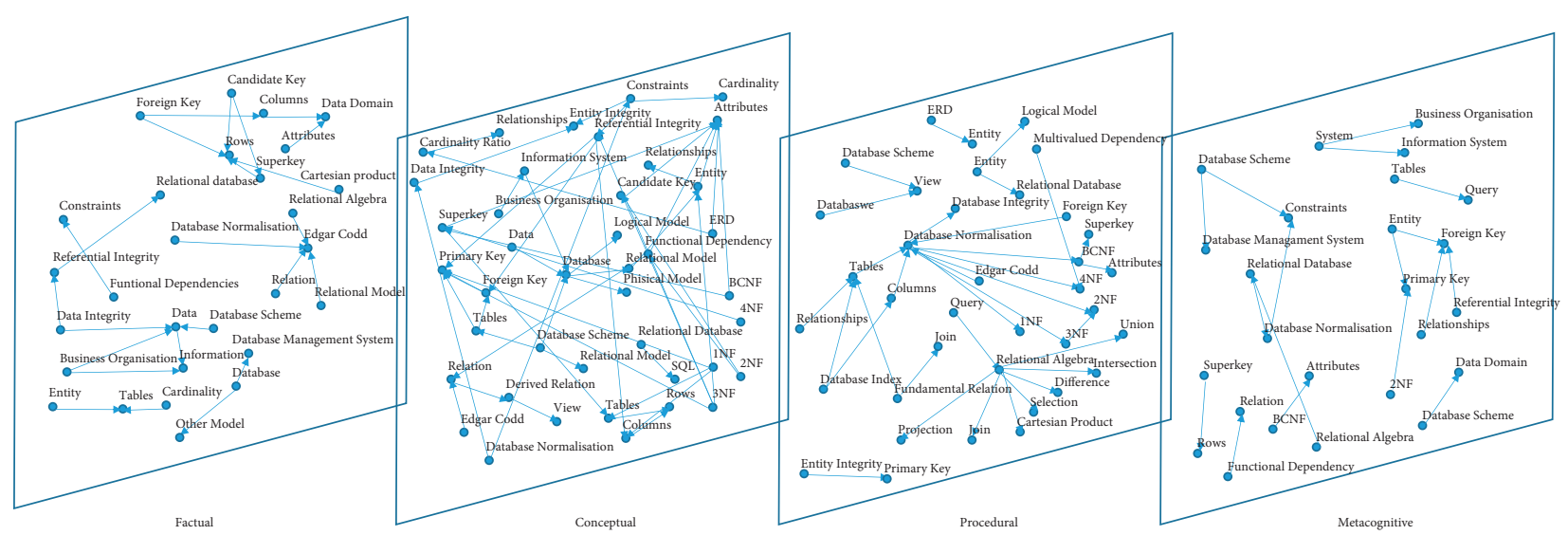

FIGURE 2: Network representation of four projected layers in a multidimensional knowledge network (MKN) for the learning outcomes of the database domain.

TABLE 1: Values of average degree, average weighted degree, network diameter, average path length, average clustering coefficient, graph density and the number of connected components W/S (weakly/strongly) for the projection layers of $i p(M)$ and $m p(M)$.

\begin{tabular}{lccccccc}
\hline Layer & $\begin{array}{c}\text { Average } \\
\text { degree }\end{array}$ & $\begin{array}{c}\text { Average } \\
\text { weighted } \\
\text { degree }\end{array}$ & $\begin{array}{c}\text { Network } \\
\text { diameter }\end{array}$ & $\begin{array}{c}\text { Average } \\
\text { path } \\
\text { length }\end{array}$ & $\begin{array}{c}\text { Average } \\
\text { clustering } \\
\text { coefficient }\end{array}$ & $\begin{array}{c}\text { Network } \\
\text { density }\end{array}$ & $\begin{array}{c}\text { Connected } \\
\text { components W/S }\end{array}$ \\
\hline Factual & 0.322 & 0.881 & 3 & 1.32 & 0.014 & 0.006 & $41 / 59$ \\
Conceptual & 0.966 & 3.068 & 8 & 2.608 & 0.017 & 0.017 & $17 / 59$ \\
Procedural & 0.847 & 3.119 & 6 & 2.769 & 0.015 & 0.015 & $20 / 56$ \\
Metacognitive & 0.339 & 1.220 & 3 & 1.433 & - & 0.006 & $39 / 59$ \\
Monolayer & 2.441 & 8.288 & 10 & 4.051 & 0.096 & 0.042 & $1 / 23$ \\
\hline
\end{tabular}

high fragmentation of basic constituents needed for knowledge acquisition.

The primary aim of any centrality measure is the ranking of the nodes for producing an ordered list of the nodes according to their relevance in the structure [19, 21, 47, 48, 52]. The high degree centrality is inherent for hub nodes: in the studied case, the degree reflects how influential (central) is a concept for the process of knowledge acquisition (Table 2).

At the monolayer, according to the unweighted and weighted variants, we obtain one shared concept of tables, and at a procedural layer, the concept of database normalization, which is the core procedure during the design and construction of databases, and at metacognitive layer, we obtain the concepts of database_scheme (unweighted) and query (for weighted variant). This makes a sense, knowing that querying is the primary programming abstraction of the relational databases, and database_scheme makes a blueprint of the database construction.

Still, despite different rankings of the top concepts, we have received the valuable set of concepts characterized by the rich content. These concepts acquire knowledge of a broader scope of concepts (supported by many previous nodes) and result in a higher cognitive process dimension. Nodes with low in-degree represent concepts which can be starting points of the navigational path through the content, and vice versa; nodes with high out-degree correspond to concepts with learning outcomes of a higher level of the cognitive dimensions. For instance, it can be noticed that concepts Table and Database normalization are detected as hubs and are directly linked with the level of cognitive load in an interactive learning setting, which can be cognitively challenging for a novice learner because of a high level of transitivity between knowledge dimensions. Hence, it would be advisable to plan the acquisition of these concepts earlier rather than later in the instruction (navigational) plan.

Next, we perform an analysis of degree values for the top 20 ranked nodes in $\mathrm{MKN}$, as reported in Figure 3. It is evident that the node degree measure acts similarly in all layers and holds similar for in-degree, out-degree, and strength as well. Node degree in MKN layers exhibits similar results as in the monolayer network. Concepts (nodes) labeled as Table, Database normalization, Attributes, and Database scheme have high values of degree centrality, indicating that those nodes require additional effort in cognitive processing at a certain level of knowledge (as already noticed above). These nodes are supported with several lower-level nodes, indicating a more complex knowledge structure and necessity of the existence of student's prior knowledge. Considering the role of network structure and different knowledge dimensions as relationship types, the influence of degree can be significant in the form of interplay between the cognitive process dimension, knowledge type, and instruction. However, since the node degree in a multilayer network is a vector, aggregation of measures could indicate which nodes are more influential and how are related among different layers.

Figure 4 visualizes nodes of the monolayer network in different colors according to its degree values. Similar visualizations for closeness centrality, betweenness, and eccentricity in a monolayer network are reported in 
TABLE 2: Top five highly ranked nodes according to the degree $\left(d c_{i}\right)$, in-degree $d c_{i}^{\text {in }}$, and out-degree $d c_{i}^{\text {out }}$ values in a monolayer network.

\begin{tabular}{|c|c|c|c|c|c|c|}
\hline & Node & $d c_{i}$ & Node & $d c_{i}^{\text {in }}$ & Node & $d c_{i}^{\text {out }}$ \\
\hline \multirow{5}{*}{ Monolayer } & Tables & 14 & Primary_key & 10 & Relational_algebra & 9 \\
\hline & Database_normalization & 12 & Attributes & 9 & Database_normalization & 8 \\
\hline & Database_scheme & 11 & Tables & 8 & Database_scheme & 7 \\
\hline & Relational_algebra & 11 & Relation & 7 & Entity & 7 \\
\hline & Primary_key & 10 & Rows & 7 & Tables & 6 \\
\hline \multirow{5}{*}{ Factual } & Database_scheme & 3 & Database_scheme & 3 & Business_organisation & 3 \\
\hline & Tables & 3 & Tables & 3 & Data & 2 \\
\hline & Data & 3 & Information & 2 & Constraints & 1 \\
\hline & Business_organisation & 3 & Relational_database & 2 & Database & 1 \\
\hline & Database & 2 & Data & 1 & Referential_integrity & 1 \\
\hline \multirow{5}{*}{ Conceptual } & Attributes & 7 & Attributes & 6 & Database & 5 \\
\hline & Database & 6 & Rows & 5 & Tables & 3 \\
\hline & Tables & 6 & Primary_key & 5 & Foreign_key & 3 \\
\hline & Columns & 6 & Columns & 4 & SQL & 3 \\
\hline & Foreign_key & 5 & Data_domain & 3 & Referential_integrity & 3 \\
\hline \multirow{5}{*}{ Procedural } & Database_normalization & 11 & Relation & 5 & Database_normalization & 7 \\
\hline & Relational_algebra & 7 & Database_normalization & 4 & Relational_algebra & 7 \\
\hline & $3 \mathrm{NF}$ & 6 & Primary_key & 3 & $3 \mathrm{NF}$ & 5 \\
\hline & Relation & 6 & Entity & 2 & Entity & 2 \\
\hline & Entity & 4 & Tables & 2 & Tables & 2 \\
\hline \multirow{5}{*}{ Metacognitive } & Database_scheme & 3 & Foreign_key & 3 & Database_scheme & 2 \\
\hline & Foreign_key & 3 & Attributes & 2 & $2 \mathrm{NF}$ & 2 \\
\hline & $2 \mathrm{NF}$ & 2 & Primary_key & 2 & Entity & 2 \\
\hline & Attributes & 2 & Rows & 2 & Selection & 2 \\
\hline & Constraints & 2 & Database_scheme & 1 & Constraints & 1 \\
\hline
\end{tabular}

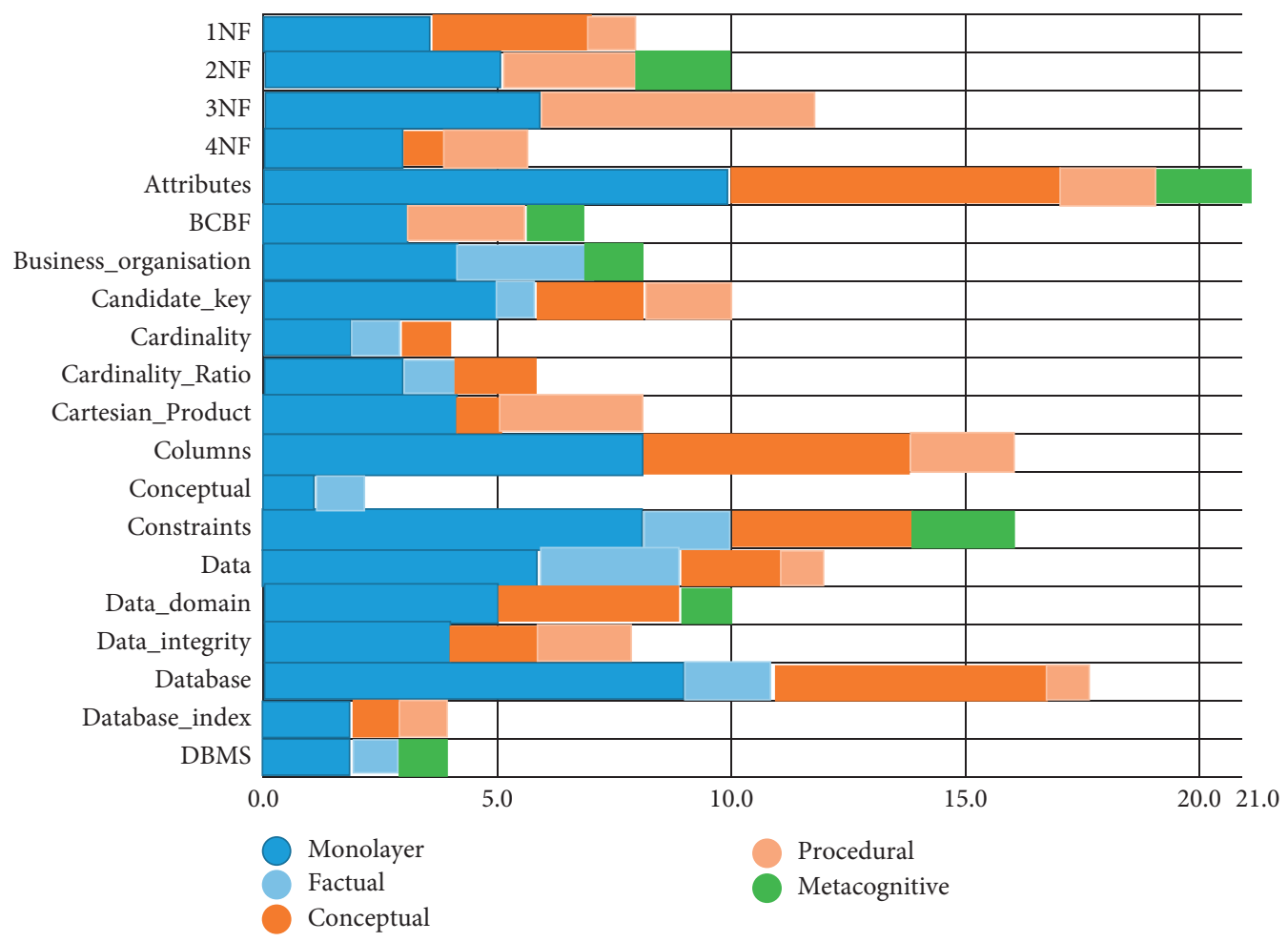

FIGURE 3: Diagnostic analysis of degree measure in MKN shows relations across layers.

Supplementary Materials. The figure suggests that the monolayer network exhibits some hierarchical properties that may be analyzed on the meso-scale level.
Analysis of weighted degree (weighted in-degree and weighted out-degree) does not show substantial differences between the top-ranked nodes across layers. This implies 




Figure 4: The visualization of the monolayer network according to the degree. Nodes with high degree values are darker, while nodes with lower degree values are lighter colored.

that although the same nodes appear at different knowledge levels, their cognitive complexity can vary due to the knowledge dimension overlap. In practice, these overlap ladders of the cognitive domain and the knowledge dimension do have limits and need interpretations. The illustrative example is "Is it better for a student to have achieved factual knowledge of creating rather than metacognitive knowledge of remembering?" There is no single answer since it differs depending on the teaching style and subject area.

Still, the top degree lists (concepts) differ substantially, meaning that the identification of the most essential (highly ranked) concepts highly depends on the variant of the degree centrality measure with in- and out-variants and used weighting. This is an indication that, for better identification of the most influential concepts, we should opt for more sophisticated insights; so first, we proceed with the quantification of centrality measures (Table 3).

Table 4 presents the values of closeness centrality, betweenness centrality, and eccentricity in a monolayer network. Closeness centrality quantifies how close a node is to all other nodes in the network: the smaller the total distance from a node $v$ to all other nodes, the more important the node $v$ [21]. According to the closeness centrality values, entity integrity is a top-ranked node followed by four nodes with values of the same range. This implies that these four concepts should be considered as possible starting points in a learning navigation path since their closeness values correspond with high degree values. Nodes that take a starting point role in learning navigational paths are crucial for effective knowledge acquisition. The research reported in
[46] also emphasizes the importance of the closeness centrality since it operationalizes the structural relevance in the knowledge representation. Note that all nodes with closeness centrality equal to 1 refer to nodes that belong to small disconnected components usually composed of two nodes, which also indicates the fragmentation of knowledge.

Nodes with high betweenness in Table 4 are Database normalization and Tables, followed by Constraints and Relational algebra. It is known that these nodes take a bridging role in the network; i.e., they are in charge of the information flow-either for describing other knowledge units (the incoming edges) or for influencing other knowledge units (the outgoing edges). These nodes are of high importance and serve as the glue in the knowledge representation model.

The eccentricity aims to determine a node that minimizes the maximum distance to any other node in the graph. In other words, eccentricity quantifies the distance between the concepts. Top-ranked Selection, Attributes, Derived relation, and System represent core concepts needed for acquiring more complex knowledge. The high value of eccentricity indicates that concept could be essential in minimizing learning effort while acquiring more complex concepts. Except for the most essential concept of Database at the factual layer, the centrality analysis was failing to reveal better structural ordering or sequencing of the concepts during learning.

Detecting communities in complex networks is of particular interest when identifying nodes that share properties and dynamics [21]. In this research, we apply the Louvain algorithm [62] for community detection, and Figure 5 shows 
TABLE 3: Top five highly ranked nodes according to the weighted degree $\left(o_{i j}\right)$, weighted in-degree $\left(o_{i j}^{\text {in }}\right)$, and weighted out-degree $\left(o_{i j}^{\text {out }}\right)$ in a weighted monolayer network.

\begin{tabular}{|c|c|c|c|c|c|c|}
\hline & Node & $o_{i j}$ & Node & $o_{i j}^{\text {in }}$ & Node & $o_{i j}^{\text {out }}$ \\
\hline \multirow{5}{*}{ Monolayer } & Tables & 62 & Tables & 34 & Database_normalization & 41 \\
\hline & Database_normalization & 57 & Primary_key & 32 & Tables & 28 \\
\hline & Relation & 41 & Relation & 31 & Entity & 25 \\
\hline & Foreign_key & 38 & Attributes & 27 & Database_scheme & 23 \\
\hline & Entity & 38 & Columns & 24 & Database & 23 \\
\hline \multirow{5}{*}{ Factual } & Database_scheme & 12 & Database_scheme & 12 & Data & 6 \\
\hline & Tables & 10 & Tables & 10 & Business_organisation & 6 \\
\hline & Data & 8 & Information & 6 & Database & 4 \\
\hline & Business_organisation & 6 & Database_ManagementSystem & 4 & Candidate_key & 4 \\
\hline & Database & 6 & Relationships & 4 & Cardinality & 4 \\
\hline \multirow{5}{*}{ Conceptual } & Tables & 29 & Columns & 18 & Database & 19 \\
\hline & Columns & 24 & Primary_key & 18 & Tables & 14 \\
\hline & Database & 23 & Attributes & 16 & Referential_integrity & 12 \\
\hline & Foreign_key & 20 & Tables & 15 & $1 \mathrm{NF}$ & 12 \\
\hline & Attributes & 18 & Rows & 12 & Database_scheme & 11 \\
\hline \multirow{5}{*}{ Procedural } & Database_normalization & 52 & Relation & 22 & Database_normalization & 36 \\
\hline & Relation & 26 & Database_normalization & 16 & Relational_algebra & 14 \\
\hline & $3 \mathrm{NF}$ & 17 & Tables & 9 & $3 \mathrm{NF}$ & 11 \\
\hline & Tables & 17 & $2 \mathrm{NF}$ & 8 & Projection & 11 \\
\hline & Entity & 16 & Join & 8 & Entity & 9 \\
\hline \multirow{5}{*}{ Metacognitive } & Foreign_key & 12 & Foreign_key & 12 & $2 \mathrm{NF}$ & 7 \\
\hline & Query & 11 & Query & 6 & Business_organisation & 6 \\
\hline & Relational_algebra & 10 & System & 6 & Entity & 6 \\
\hline & Functional_dependency & 9 & Attributes & 6 & Tables & 6 \\
\hline & Constraints & 8 & Primary_key & 6 & Query & 5 \\
\hline
\end{tabular}

TABLE 4: Top five highly ranked nodes (concepts) according to the closeness centrality $\left(c c_{i}\right)$, betweenness centrality $\left(b c_{i}\right)$, and eccentricity $\left(C_{e e c}\right)$ in a monolayer network.

\begin{tabular}{|c|c|c|c|c|c|c|}
\hline & Node & $c c_{i}$ & Node & $b c_{i}$ & Node & $C_{e e c}$ \\
\hline \multirow{5}{*}{ Monolayer } & Entity integrity & 1.0 & Database normalization & 806.546 & Selection & 10.0 \\
\hline & Database scheme & 0.362 & Tables & 624.485 & Attributes & 9.0 \\
\hline & Database normalization & 0.359 & Constraints & 588.1 & Derived_relation & 9.0 \\
\hline & Tables & 0.354 & Relational_algebra & 507.413 & System & 8.0 \\
\hline & Relational algebra & 0.336 & Query & 490.413 & Business_organisation & 8.0 \\
\hline \multirow{5}{*}{ Factual } & Database & 1.0 & Database & 2.0 & Business_organisation & 3.0 \\
\hline & Referential_integrity & 1.0 & Referential_integrity & 2.0 & Functional_dependency & 3.0 \\
\hline & Candidate_key & 1.0 & Data & 2.0 & Data & 2.0 \\
\hline & Cardinality & 1.0 & Constraints & 2.0 & Constraints & 2.0 \\
\hline & Cardinality_Ratio & 1.0 & Candidate_key & 0.0 & Database & 1.0 \\
\hline \multirow{5}{*}{ Conceptual } & View & 1.0 & Data_domain & 64.0 & Information_System & 8.0 \\
\hline & Entity_integrity & 1.0 & Constraints & 55.0 & Database & 7.0 \\
\hline & Cartesian_product & 1.0 & Attributes & 39.5 & Relational_model & 6.0 \\
\hline & Relational_algebra & 1.0 & Columns & 28.5 & Database_scheme & 6.0 \\
\hline & SQL & 0.8 & Entity & 25.0 & Entity & 5.0 \\
\hline \multirow{5}{*}{ Procedural } & Database_scheme & 1.0 & Database_normalization & 156.0 & Relational_algebra & 6.0 \\
\hline & Relationships & 1.0 & $3 \mathrm{NF}$ & 49.5 & $3 \mathrm{NF}$ & 5.0 \\
\hline & Fundamental_relation & 1.0 & Tables & 46.0 & Cartesian_product & 5.0 \\
\hline & $2 \mathrm{NF}$ & 1.0 & Entity & 43.0 & Projection & 5.0 \\
\hline & $\mathrm{BCBF}$ & 1.0 & Relational_database & 36.0 & Database_index & 5.0 \\
\hline \multirow{5}{*}{ Metacognitive } & Entity & 1.0 & Database_scheme & 4.0 & Tables & 3.0 \\
\hline & Relational_algebra & 1.0 & Constraints & 3.0 & Database_normalization & 3.0 \\
\hline & Functional_dependency & 1.0 & Relational_algebra & 2.0 & Constraints & 2.0 \\
\hline & Referential_integrity & 1.0 & Query & 2.0 & Query & 2.0 \\
\hline & Database_scheme & 1.0 & Functional_dependency & 1.0 & $2 \mathrm{NF}$ & 2.0 \\
\hline
\end{tabular}




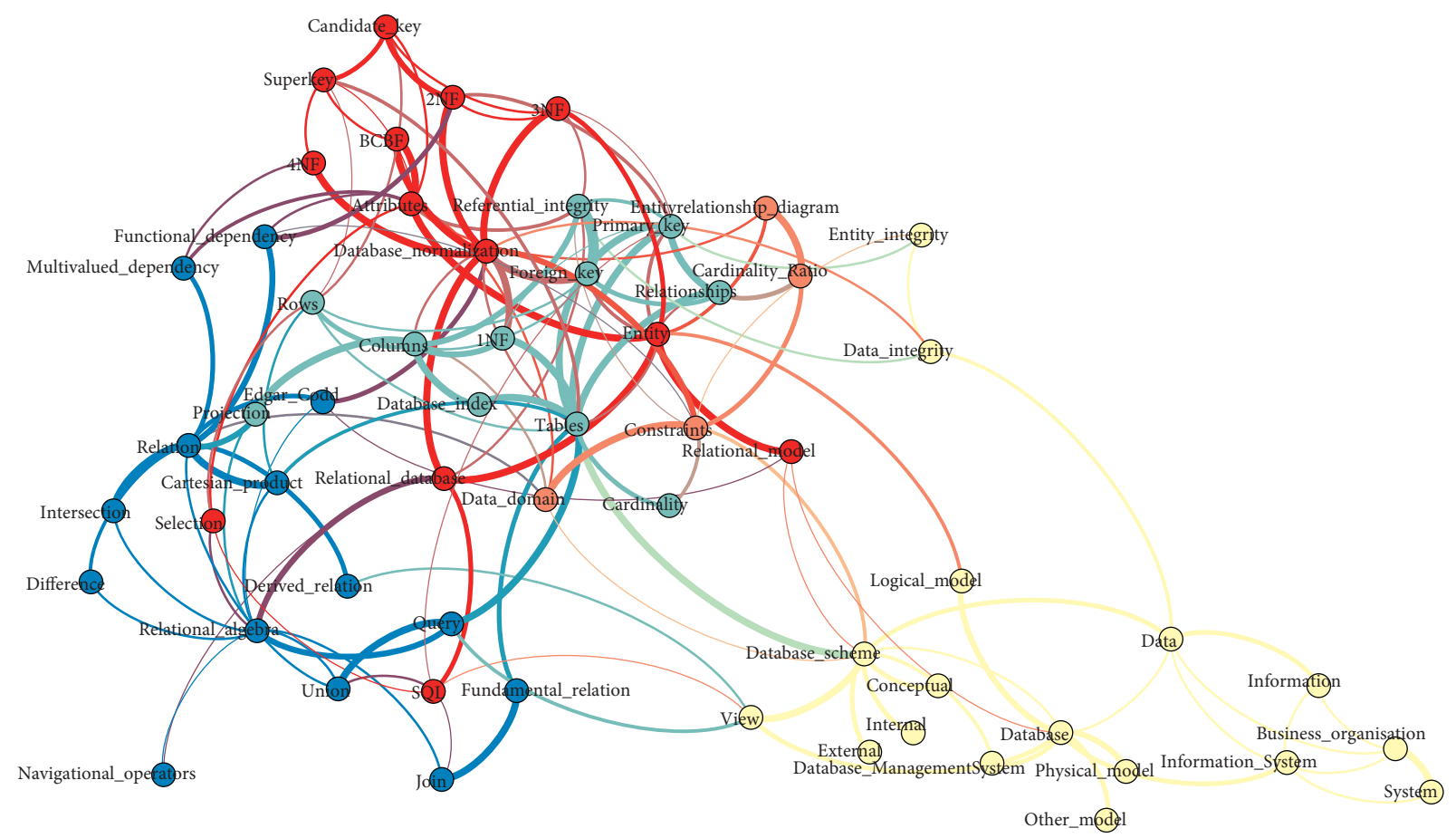

FIgURE 5: The structure of six communities in a monolayer MKN network

the results of community detection in the monolayer MKN network.

There are six communities in the monolayer network, while the whole MKN is dispersed into 22 communities. According to the community structure in the monolayer network (Figure 5), we assume that closely related learning concepts belong to the same community, forming a coherent group of knowledge units. For example, concepts Database, Database Management System, Logical Model, and Physical Model while Relational Algebra, Query, and Union belong to another community. Still, the community structure is not ideally discovered, since Database Normalization, 2NF, 3NF, and $4 \mathrm{NF}$ are in the same community, while $1 \mathrm{NF}$ is in the other, although semantically belongs to the same. However, it seems that community structure provides a good insight into how concepts are clustered into more complex units and is of benefit for the detection of coherent groups of concepts which can be organized in the same learning units (lectures).

5.2. Analysis of Correlations and Overlapping between $M K N$ Layers. A standard way to quantify the presence of interlayer degree correlations is to calculate Pearson's and Spearman's interlayer correlation coefficients for indication of how degree sequences of two layers are correlated [10]. Networks that are degree assortative or degree disassortative have higher information content than networks that are degree nonassortative [61]. The values of assortativity are obtained as Pearson and Spearman pairwise correlation coefficients for multilayer networks, as shown in Tables 5 and 6, respectively. Each of those two coefficients exposes slightly different behaviors. More precisely, the values of
TABLE 5: Interlayer assortativity: Pearson's correlation coefficients.

\begin{tabular}{lcccc}
\hline Layer & Factual & Conceptual & Procedural & Metacognitive \\
\hline Factual & 1 & 0.096 & -0.175 & 0.111 \\
Conceptual & 0.096 & 1 & -0.033 & 0.281 \\
Procedural & -0.175 & -0.033 & 1 & 0.155 \\
Metacognitive & 0.111 & 0.281 & 0.155 & 1 \\
\hline
\end{tabular}

TABLE 6: Interlayer assortativity: Spearman's correlation coefficients.

\begin{tabular}{lcccc}
\hline Layer & Factual & Conceptual & Procedural & Metacognitive \\
\hline Factual & 1 & 0.096 & -0.241 & 0.085 \\
Conceptual & 0.042 & 1 & 0.023 & 0.226 \\
Procedural & -0.241 & 0.023 & 1 & 0.16 \\
Metacognitive & 0.085 & 0.226 & 0.16 & 1 \\
\hline
\end{tabular}

Pearson's correlation coefficient indicate disassortativity of factual, conceptual, and procedural knowledge. The reason stems from the very type of knowledge they represent and their differentiation: factual knowledge represents specific bits of information; conceptual knowledge includes more complex organized knowledge (schemas, models, and theories), and procedural knowledge reflects knowledge of "processes." In order to acquire knowledge of a higher level, "the deeper understanding" (higher cognitive process dimension) of low-level units is crucial, hence the disassortativity between layers.

Layers of the multidimensional knowledge network (MKN) stem from four knowledge dimensions. They are sequenced from the detailed factual knowledge in the factual layer to the abstract metacognitive knowledge in the 
TABLE 7: The percentage overlapping of nodes in the MKN network

\begin{tabular}{lcccc}
\hline Layer & Factual & Conceptual & Procedural & Metacognitive \\
\hline Factual & \multirow{2}{*}{100} & 30.5 & 23.7 & 20.3 \\
Conceptual & & 100 & 52.5 & 32.2 \\
Procedural & & & 100 & 30.5 \\
Metacognitive & & & & 100 \\
\hline
\end{tabular}

metacognitive layer. In some cases, edges of the layers are not mutually exclusive, which can be indicated by the nodes overlapping values quantified by correlations between the degrees of the same node at different layers. On the other side, edge overlapping also suggests that the relation between two nodes might be shared by more than one layer, which could indicate redundancy of information input. In Tables 7 and 8 , we show the percentage of overlapping for nodes and edges, respectively. The results confirm the organization of the layers according to Bloom's taxonomy is highly advisable since edge overlapping between conceptual and metacognitive is minimal (below 3.2\%), while the node overlap can go to the high $52.5 \%$.

Table 9 shows the Frobenious distance used to quantify layer distance in terms of paths. The highest values of Frobenious distance are achieved for metacognitive/factual layers' pairs, which are the most distant layers. This is an indication of higher cognitive effort while traversing from factual to metacognitive layer-a larger knowledge "jump" during learning. In other words, learning "fragments of information" (factual knowledge) requires establishing connections between fragmented facts and applying them in new situations, which require a higher level of students' cognition (metacognitive knowledge). One of the directions for the reduction of the complexity of the proposed MKN model, especially in less distant layers (factual, conceptual, and procedural), can be achieved by structural reducibility proposed in [63].

Still, this remains an open challenge in future research plans. The results obtained from MKN analysis can shed light on the causes of increased cognitive demands, indicate vulnerabilities in the knowledge (more specific and identify knowledge units that require modification of instructional strategies), and consequently guide the design and optimization of learning outcomes. Therefore, the relationship between the structure of information and external representation of knowledge should be pursuit with the identification of concepts that play the key role of "basic building blocks"- high in-degree nodes in monolayer network and comparison with results generated from projected $\mathrm{MKN}$; the detection of concepts with high betweenness to reveal the glue concepts of the domain represented in MKN and with high value of eccentricity to detect concepts that could be essential in minimizing the learning effort while acquiring more complex concepts; the identification of clusters of concepts-communities can lead to better planning of the lectures; and understanding of the knowledge gap between cognitive layers can reduce the overload burden from the novice in the field.

In general, the level of knowledge dimension that is selected for external representation can influence the
TABLE 8: The percentage overlapping of edges in the MKN network.

\begin{tabular}{lcccc}
\hline Layer & Factual & Conceptual & Procedural & Metacognitive \\
\hline Factual & 100 & 0 & 0 & 0 \\
Conceptual & & 100 & 0 & 3.2 \\
Procedural & & & 100 & 1.6 \\
Metacognitive & & & & 100 \\
\hline
\end{tabular}

TABLE 9: Frobenious distance calculated between all pairs of nodes in each layer separately.

\begin{tabular}{lcccc}
\hline Layer & Factual & Conceptual & Procedural & Metacognitive \\
\hline Factual & - & 0 & 0.151 & 0.897 \\
Conceptual & & - & 0.144 & 0.019 \\
Procedural & & & - & 0.192 \\
Metacognitive & & & & - \\
\hline
\end{tabular}

adoption of information, including the activation of the cognitive process. With an inadequate representational model, the learning process can be impeded by avoiding the coherent knowledge units, which in turn can lead to structural vulnerability of the domain model during the knowledge acquisition.

\section{Conclusion}

In the discipline of technology-enabled learning in general, one of the most challenging problems is the study of the formation and representation of knowledge structures during learning. The goal is often accomplished by expressing the expert's (tutor) knowledge, which is presumed to be well organized, coherent, and consisting of rich expertise about the subject [52]. Recent cognitively oriented research on learning implicates that there is a close relationship between knowledge structure and its content in interaction with the cognitive architecture and learner's ability to process that information. Hence, this supports the idea that the knowledge system is an interwoven cohesive network that differs according to one's mental model, prior knowledge, and preferences. Understanding the structure of scientific knowledge often refers to topological features where coherence and contingency have a high correlation with crucial concepts and their interconnectedness. Thus, Koponen and Nousiainen in [1] emphasized to make coherence a clear and useful notion and to design educational solutions; there must be a chain of connections from coherence to the operational measures used to characterize knowledge networks. Siew in [50] used macrolevel network measures to quantify the structure of a monolayer network of concept for the domain of psychology, trying to identify and prioritize the "glue" concepts in the network and showing that internal representation of the students' knowledge map can be an indicator of expected performance and specifically inherent to various learning styles. Hence, the progress can be achieved by better personalization of the content. In this study, we have reached a step further and proposed a multilayered organization of external knowledge as a representation modeling alternative. To this end, we 
proposed modeling according to the revised Bloom's taxonomy in a multidimensional knowledge network (MKN).

Recently, some advances toward shedding more light on this ambitious pursuit paw the way of the future research quests. Several studies indicate that multilayer or multiplex representations are adequate modeling approaches for the cognitive representation models. Stella in [45] suggests that global and multilevel representation of the mental lexicon for acquiring vocabulary in the early stage of learning language competencies better models and quantifies the flow of information, especially emphasizing the importance of closeness centrality for spreading of activation patterns. Hence, studying the dynamics of knowledge acquisition in an e-learning system can be of utmost importance. de Arruda et al. in [64] already revealed that using the true selfavoiding random walk can efficiently model the dynamics of the knowledge acquisition, which is specifically placed at the core of the network.

Guided by findings in studies of concept and cognitive networks $[1-4,6,8,13,14,22,36,37,39,41$, $46,49,50,52,59,64,65]$, we have applied the concept mapping method to represent a knowledge system of the Database domain as the complex network. Specifically, we propose a multidimensional knowledge network (MKN) based on the multilayer network where each layer constitutes factual, conceptual, procedural, or metacognitive knowledge. In the layer, nodes are concepts or knowledge units, and the edges are weighted with regard to the revised Bloom's cognitive learning level. Additionally, we introduced two projections of $M$ : the interlayer projection and monolayer projections. The proposed interlayer projection is contrasted with monolayer projection by comparing characterizations of the centrality measures: degree centrality, closeness centrality, betweenness centrality, and eccentricity. The study revealed indications of how concepts, supported with the higher number of previously introduced concepts, have a dominant role in knowledge acquisition, from a view of knowledge structure and content. This can be of use for better planning and organization of the content in the e-learning system, uniquely when equipped together with continuous evaluation of students' progress, which can lead to a better adaptation of the system.

Moreover, obtained results indicate that $\mathrm{MKN}$ is the adequate model to study the importance and groupings of the concept, aiming toward the more efficient organization of concepts. Our study indicates that the principles of the knowledge organization of concepts enabling the detection of ones that are candidates for entry points of the navigational paths or the ones which acquire a higher level of the cognitive domain, hence the ones that are crucial for reducing or increasing a cognitive load during learning. These features were also recognized by [51], whose key concepts were central from the viewpoint of the richness of subject content and correlated with learning gains as well. An important, but as of yet, incompletely resolved issue is how the construction of knowledge network could directly influence the efficiency of navigation paths during learning, especially with regard to acquiring knowledge on the highest level of complexity.
Considering the importance of the study of the complex network for understanding and simulating cognitive processes, the correlations between knowledge dimensions were investigated. Although there is a substantial similarity regarding key concepts in the monolayer network, the assortativity and shortest path distance values could indicate elements of knowledge structures that learners can activate rapidly and apply to improve the students' knowledge. This certainly depends on the mental models of individuals, their prior knowledge, cognitive functioning (interaction of working memory and long-term memory systems), as well as navigational patterns, learning styles, and preferences. The obtained results suggest that careful modeling brings different perspectives onto modeling of the external knowledge and results in a more comprehensive understanding of how the knowledge should be organized across different levels of cognitive load. This is in line with findings reported in [36] where the authors emphasized the need for multiplex over monolayer representation for early language learning since it allows for quantification of distinct phases in the process.

The analysis of the factual layer provides quantification for the well-studied fragmentation problem (lack of established connections between facts into a more extensive system of domain knowledge $[15,38]$ by calculating the network measures). This is a step toward bridging the gap between the fragmentation of factual knowledge and a more in-depth level or integration or systematic organization of domain knowledge in adaptive e-learning systems. Apart from the knowledge of different strategies and knowledge of cognitive tasks, metacognitive knowledge also includes a self-awareness of proficiency in the domain. Hence, without the self-awareness of lack at any of factual, conceptual, or procedural layers, it is unlikely that students will make any progress in acquiring or constructing additional knowledge.

In this study, we reach for a better representation of the external knowledge, resulting in the more comprehensive insights on how the knowledge should be organized across different levels of cognitive load. To this end, we proposed modeling according to the revised Bloom's taxonomy. We have confirmed that careful modeling shed different perspectives onto modeling of the external knowledge representation. Still, there remains the open challenge of how we should approach to modeling and quantification of internal student's model of the domain.

Despite the promising results of the $\mathrm{MKN}$, there are still many open research questions, which we plan to address in future research. Specifically, this includes the application of the obtained results into the e-learning system, which adapts to student's current level of knowledge, and suggests the best navigational path through the learning content and quantification of the student's progress. Additionally, we should opt for the reduction of the complexity of the proposed MKN model, where structural reducibility proposed in [63] can serve as the starting point.

\section{Data Availability}

The data used to support the findings of this study are available from the corresponding author upon request. 


\section{Conflicts of Interest}

The authors declare that there are no conflicts of interest regarding the publication of this paper.

\section{Acknowledgments}

This work has been supported in part by the University of Rijeka under the project numbers uniri-drustv-18-20 and uniri-drustv-18-38.

\section{Supplementary Materials}

Supplementary Text 1: network structure analysis on the global and local level. Supplementary Figure S1: the visualization of the monolayer network after applying Force Atlas layout algorithm. Supplementary Figure S2: visualization of closeness centrality in a monolayer network. Nodes with high closeness values are colored darker, whilst those with lower values are lighter. Supplementary Figure S3: visualization of betweenness centrality in a monolayer network. Nodes with high betweenness values are darker, whilst those with lower values are lighter. Supplementary Figure S4: visualization of eccentricity in a monolayer network. Nodes with high eccentricity values are darker blue, whilst those with lower values are brown. (Supplementary Materials)

\section{References}

[1] I. T. Koponen and M. Pehkonen, "Coherent knowledge structures of physics represented as concept networks in teacher education," Science \& Education, vol. 19, no. 3, pp. 259-282, 2010.

[2] I. T. Koponen and M. Nousiainen, "Modelling students' knowledge organisation: genealogical conceptual networks," Physica A: Statistical Mechanics and Its Applications, vol. 495, pp. 405-417, 2018.

[3] I. Koponen and M. Nousiainen, "Pre-service physics teachers' understanding of the relational structure of physics concepts: organising subject contents for purposes of teaching," International Journal of Science and Mathematics Education, vol. 11, no. 2, pp. 325-357, 2013.

[4] I. T. Koponen, T. Kokkonen, and M. Nousiainen, "Dynamic systems view of learning a three-tiered theory in physics: robust learning outcomes as attractors," Complexity, vol. 21, no. S2, pp. 259-267, 2016.

[5] A. Cañas, J. Coffey, M. Carnot, and P. J. Feltovich, “A summary of literature pertaining to the use of concept mapping techniques and technologies for education and performance support," Technical Report, Chief of Naval Education and Training, Pensacola, FL, USA, 2003.

[6] I. T. Koponen, M. Nousiainen, and M. Nousiainen, "Concept networks in learning: finding key concepts in learners' representations of the interlinked structure of scientific knowledge," Journal of Complex Networks, vol. 2, no. 2, pp. 187-202, 2014.

[7] B. Bloom, M. Englehart, E. Furst et al., Taxonomy of Educational Objectives: The Classification of Educational Goals". Handbook I: Cognitive Domain, Longmans Green \& Co., New York, NY, USA, 1956.
[8] C. S. Q. Siew, D. U. Wulff, N. M. Beckage, and Y. N. Kenett, "Cognitive network science: a review of research on cognition through the lens of network representations, processes, and dynamics," Complexity, vol. 2019, Article ID 2108423, 24 pages, 2019.

[9] M. Nadrljanski, Đ. Vukić, and Đ. Nadrljanski, "Multi-agent systems in E-Learning," in Proceedings of the 41st International Convention on Information and Communication Technology, Electronics and Microelectronics-MIPRO, Opatija, Croatia, May 2018.

[10] R. Noldus and P. Van Mieghem, "Assortativity in complex networks," Journal of Complex Networks, vol. 3, no. 4, pp. 507-542, 2015.

[11] B. A. Schwendimann, "Concept maps as versatile tools to integrate complex ideas: from kindergarten to higher and professional education," Knowledge Management \& E-Learning: An International Journal, vol. 7, no. 1, pp. 73-99, 2015.

[12] C.S. Q. Siew and M. S. Vitevitch, "The phonographic language network: using network science to investigate the phonological and orthographic similarity structure of language," Journal of Experimental Psychology: General, vol. 148, no. 3, pp. 475-500, 2019.

[13] M. Stella, "Cohort and rhyme priming emerge from the multiplex network structure of the mental lexicon," Complexity, vol. 2018, Article ID 6438702, 14 pages, 2018.

[14] M. Stella, N. M. Beckage, M. Brede, and M. De Domenico, "Multiplex model of mental lexicon reveals explosive learning in humans," Scientific Reports, vol. 8, no. 1, p. 2259, 2018.

[15] C. Bereiter and M. Scardamalia, "Beyond bloom's taxonomy: rethinking knowledge for the knowledge age," in International Handbook of Educational Change, A. Hargreaves, A. Lieberman, M. Fullan et al., Eds., pp. 675-692, Kluwer Academic Publishers, London, UK, 1998.

[16] L. W. Anderson, D. R. Krathwohl, P. W. Airasian et al., A Taxonomy for Learning, Teaching, and Assessing: A Revision of Bloom's Taxonomy of Educational Objectives, Pearson, New York, NY, USA, 2001.

[17] A. Amer, "Reflections on Bloom's revised taxonomy," Electronic Journal of Research in Educational Psychology, vol. 4, no. 1, pp. 213-230, 2006.

[18] F. Radmehr and M. Drake, "Revised bloom's taxonomy and integral calculus: unpacking the knowledge dimension," International Journal of Mathematical Education in Science and Technology, vol. 48, no. 8, pp. 1206-1224, 2017.

[19] A.-L. Barabási and M. Pósfai, Network Science, Cambridge University Press, Cambridge, UK, 2016.

[20] F. Battiston, V. Nicosia, and V. Latora, "Metrics for the analysis of multiplex networks," Physical Review E, vol. 89, Article ID 032804, 2014.

[21] L. da F Costa, F. A. Rodrigues, G. Travieso, and P. R. Villas, "Characterization of complex networks: a survey of measurements," Advances in Physics, vol. 56, no. 1, pp. 167-242, 2007.

[22] A. Baronchelli, R. Ferrer-I-Cancho, R. Pastor-Satorras, N. Chater, and M. H. Christiansen, "Networks in cognitive science," Trends in Cognitive Sciences, vol. 17, no. 7, pp. 348-360, 2013.

[23] A. Sole, A. Arenas, and S. Gomez, "Effect of shortest path multiplicity on congestion of multiplex networks," New Journal of Physics, vol. 21, no. 3, Article ID 035003, 2019.

[24] J. Cardillo, M. Gómez-Gardeñes, M. Zanin et al., "Emergence of network features from multiplexity," Scientific Reports, vol. 3, no. 1, p. 1344, 2013. 
[25] J. F. Donges, H. C. H. Schultz, N. Marwan, Y. Zou, and J. Kurths, "Investigating the topology of interacting networks," The European Physical Journal B, vol. 84, no. 4, pp. 635-651, 2011.

[26] J. Gao, D. Li, and S. Havlin, "From a single network to a network of networks," National Science Review, vol. 1, no. 3, pp. 346-356, 2014.

[27] L. da Fontoura Costa, "Learning about knowledge: a complex network approach," Physical Review E, vol. 74, no. 2, Article ID 026103, 2006.

[28] M. Berlingerio, M. Coscia, F. Giannotti, A. Monreale, and D. Pedreschi, "Foundations of multidimensional network analysis," in Proceedings of the Advances in Social Networks Analysis and Mining (ASONAM), vol. 485-489, Kaohsiung, Taiwan, August 2011.

[29] M. De Domenico, A. Solé- Ribalta, E. Cozzo et al., "Mathematical formulation of multilayer networks," Physical Review $X$, vol. 3, no. 4, pp. 041022-041037, 2013.

[30] M. De Domenico, M. A. Porter, and A. Arenas, "MuxViz: a tool for multilayer analysis and visualization of networks," Journal of Complex Networks, vol. 3, no. 2, pp. 159-176, 2015.

[31] M. Kivela, A. Arenas, M. Barthelemy, J. P. Gleeson, Y. Moreno, and M. A. Porter, "Multilayer networks," Journal of Complex Networks, vol. 2, no. 3, pp. 203-271, 2014.

[32] M. Kurant and P. Thiran, "Layered complex networks," Physical Review Letters, vol. 96, no. 13, pp. 138701-138705, 2006.

[33] S. O. Tergan, "Digital concept maps for managing knowledge and information," in Knowledge and Information Visualization, pp. 185-204, Springer, Berlin, Germany, 2005.

[34] S. Boccaletti, G. Bianconi, R. Criado et al., "The structure and dynamics of multilayer networks," Physics Reports, vol. 544, no. 1, pp. 1-122, 2014.

[35] S. Martinčić-Ipšić, D. Margan, and A. Meštrović, "Multilayer network of language: a unified framework for structural analysis of linguistic subsystems," Physica A: Statistical Mechanics and Its Applications, vol. 457, pp. 117-128, 2016.

[36] M. Stella, N. M. Beckage, and M. Brede, "Multiplex lexical networks reveal patterns in early word acquisition in children," Scientic Reports, vol. 7, no. 1, p. 46730, 2017.

[37] G. Rosell-Tarragó, E. Cozzo, and A. Díaz-Guilera, "A complex network framework to model cognition: unveiling correlation structures from connectivity," Complexity, vol. 2018, Article ID 1918753, 19 pages, 2018.

[38] J. D. Branslord, A. L. Brown, and R. R. Cocking, How People Leam: Brain, Mind, Experience and school, National Academy Press., Washington, DC, USA, 1999.

[39] I. T. Koponen and M. Pehkonen, "Entropy and energy in characterizing the organization of concept maps in learning science," Entropy, vol. 12, no. 7, pp. 1653-1672, 2010.

[40] F. Safayeni, N. Derbentseva, and A. J. Cañas, "A theoretical note on concepts and the need for cyclic concept maps," Journal of Research in Science Teaching, vol. 42, no. 7, pp. 741-766, 2005.

[41] N. M. Beckage and E. Colunga, "Language networks as models of cognition: understanding cognition through language," in Towards a Theoretical Framework for Analysing Complex Linguistic Networks. Understanding Complex Systems, A. Mehler, A. Lücking, S. Banisch et al., Eds., pp. 3-28, Springer, Berlin, Germany, 2016.

[42] I. Gurevych, "Using the structure of a conceptual network in computing semantic relatedness.," in Natural Language Processing-IJCNLP 2005, Lecture Notes in Computer Science,
R. Dale, KF. Wong, J. Su et al., Eds., Vol. 3651, Springer, Berlin, Germany, 2005.

[43] J. B. Batista and L. F. Costa, "Knowledge acquisition by networks of interacting agents in the presence of observation errors," Physical Review E, vol. 82, no. 1, Article ID 016103, 2010.

[44] M. S. Vitevich and N. Castro, "Using network science in the language and clinic," International Journal of Speech-Language Pathology, vol. 17, no. 1, pp. 13-25, 2015.

[45] M. Stella and Y. N. Kenett, "Viability in multiplex lexical networks and machine learning characterizes human creativity," Big Data and Cognitive Computing, vol. 3, no. 3, p. 45, 2019.

[46] M. Stella, "Modelling early word acquisition through multiplex lexical networks and machine learning," Big Data and Cognitive Computing, vol. 3, no. 1, p. 10, 2019.

[47] S. Beliga, A. Meštrović, and S. Martincic-Ipsic, "Selectivitybased keyword extraction method," International Journal on Semantic Web and Information Systems, vol. 12, pp. 1-26, 2016.

[48] S. Beliga, A. Meštrović, and S. Martincic-Ipsic, "Toward selectivity based keyword extraction for Croatian news," CEUR Workshop Proceedings, vol. 1310, 2014.

[49] I. T. Koponen and M. Nousiainen, "Lexical networks and lexicon profiles in didactical texts for science education," in Proceedings of the International Conference on Complex Networks and Their Applications, pp. 15-27, Springer, Lisbon, Portugal, December 2019.

[50] C. S. Siew, "Using network science to analyze concept maps of psychology undergraduates," Applied Cognitive Psychology, vol. 33, no. 4, pp. 662-668, 2019.

[51] E. Yli-Panula, A. Virta, and K. Merenluoto, "A Graph-theoretic perspective on the content structure of physics lessons and its relation to Student learning gains," in Learning, Teaching, and Growth into Teacherhood in the Light of SubjectDidactical Research, pp. 55-71, University of Turku, Turku, Finland, 2011.

[52] G. Scardoni and C. Laudanna, "Centralities based analysis of complex networks," in New Frontiers in Graph Theory, Y. Zhang, Ed., pp. 323-348, Intech Open, London, UK, 2012.

[53] K. M. Carley, "Network Text Analysis: the network position of concepts," in Text Analysis for the Social Sciences, C. W. Roberts, Ed., pp. 79-102, Lawrence Erlbaum Associates, Mahwah, NJ, USA, 1997.

[54] A. E. Motter, A. P. S. De Moura, Y. C. Lai, and P. Dasgupta, "Topology of the conceptual network of language," Physical Review E-Statistical Physics, Plasmas, Fluids, and Related Interdisciplinary Topics, vol. 65, no. 6, 2002.

[55] G. Bianconi, S. Dorogovtsev, and J. Mendes, "Mutually connected component of network of networks," Physical Review E, vol. 91, no. 1, Article ID 012804, 2015.

[56] E. Estrada and J. Gómez-Gardeñes, "Communicability reveals a transition to coordinated behavior in multiplex networks," Physical Review E, vol. 89, no. 4, Article ID 042819, 2014.

[57] G. Menichetti, D. Remondini, P. Panzarasa, R. Mondragón, and G. Bianconi, "Weighted multiplex networks," PLoS One, vol. 9, no. 6, Article ID e97857, 2014.

[58] J. Gao, S. V. Buldyrev, H. E. Stanley, and S. Havlin, "Networks formed from interdependent networks," Nature Physics, vol. 8, no. 1, pp. 40-48, 2012.

[59] R. E. Krathwohl, “A revision of bloom's taxonomy: an overview," Theory Into Practice, vol. 41, no. 4, pp. 213-218, 2002. 
[60] M. Bastian, S. Heymann, and M. Jacomy, "Gephi: an open source software for exploring and manipulating networks," in Proceedings of the International AAAI Conference on Weblogs and Social Media, San Jose, CA, USA, 2009.

[61] V. Nicosia and V. Latora, "Measuring and modellling correlations in multiplex networks," Physical Review E, Statistical, Nonlinear, and Soft Matter Physics, vol. 92, no. 3, Article ID 032805, 2015.

[62] V. D. Blondel, J.-L. Guillaume, R. Lambiotte, and E. Lefebvre, "Fast unfolding of communities in large networks," Journal of Statistical Mechanics: Theory and Experiment, vol. 2008, no. 10, Article ID P10008, 2008.

[63] M. De Domenico, V. Nicosia, A. Arenas, and V. Latora, "Structural reducibility of multilayer networks," Nature Communications, vol. 6, no. 1, p. 6864, 2015.

[64] H. F. de Arruda, F. N. Silva, L. d. F. Costa, and D. R. Amancio, "Knowledge acquisition: a complex networks approach," Information Sciences, vol. 421, pp. 154-166, 2017.

[65] R. Barnett, Realizing the University in an Age of Supercomplexity, Vol. 40, Society for Research into Higher Education \& Open University Press, Philadelphia, PA, USA, 2000. 